\title{
Did the Universe Experienced a Pressure non-Crushing Type Cosmological Singularity in the Recent Past?
}

\author{
S.D. Odintsov, ${ }^{1,2,3}$ V.K. Oikonomou, ${ }^{4,5}$ \\ 1) ICREA, Passeig Luis Companys, 23, 08010 Barcelona, Spain \\ 2) Institute of Space Sciences (ICE,CSIC) C. Can Magrans s/n, 08193 Barcelona, Spain \\ 3) Institute of Space Sciences of Catalonia (IEEC), Barcelona, Spain \\ 4) Department of Physics, Aristotle University of Thessaloniki, Thessaloniki 54124, Greece \\ ${ }^{5)}$ Laboratory for Theoretical Cosmology, International Center of Gravity and Cosmos, \\ Tomsk State University of Control Systems and Radioelectronics (TUSUR), 634050 Tomsk, Russia
}

In the recent literature it has been shown that the $H_{0}$ tension may be eliminated if an abrupt physics transition changed the Cepheid parameters in the near past of the Universe, nearly $70-$ 150 Myrs ago. In this letter we stress the possibility that this abrupt transition was caused by the smooth passage of our Universe through a pressure finite-time cosmological singularity. Being a non-crushing type singularity the pressure singularity can leave its imprints in the Universe, since it occurs globally and literally everywhere. We discuss how this scenario could easily be realized by $F(R)$ gravity, with the strong energy conditions being satisfied without the need for a scalar field or specific matter fluids. We also stress the fact that the pressure singularity can affect the effective gravitational constant of $F(R)$ gravity. Moreover, we stress the fact that pressure singularities can disrupt the trajectories of bound objects in the Universe, which is also pointed out in the literature, even in the context of general relativity. We also show numerically in a general relativistic framework that elliptic trajectories are distorted and changed to different elliptic trajectories when the Universe passes through the pressure singularity. Such a disruption of the trajectories could have tidal effects on the surface of the earth, for example on sea waters and oceans, regarding the distortion of moon's elliptic trajectory. Accordingly, the distortion of earth's trajectory around the sun could have affected climatologically earth $70-150$ Myrs ago.

PACS numbers:

\section{INTRODUCTION}

The $H_{0}$ tension [1, 2] is an undoubtable fact according to the latest observations. Such discrepancy between the Cosmic Microwave Background measurements and the low-redshift sources on the present day $H_{0}$ could be a calibration argument, as was pointed out in Refs. [3, 4]. As was pointed out in Refs. [4, 5], the $H_{0}$ tension is eliminated if an abrupt transition in the Cepheid parameters have occurred before $70-150$ Myrs. Such a change could have occurred if the physics of the Universe globally changed around $70-150 \mathrm{Myrs}$ ago, and this could also have affected the strength of the gravitational force during the same period of time. In this letter we aim to discuss the possibility that such a physics change may have been caused by the smooth passage of the Universe through a pressure singularity. Pressure singularities are mild non-crushing type singularities, which also respect the strong energy conditions. It is known that such singularities cause deformations in the trajectories of bound systems [6]. Thus, if the Universe passed through a pressure singularity before $70-150 \mathrm{Myrs}$, it is possible that the trajectories of the bound planets in the solar system could also have been affected. In addition the orbit of the moon around earth could have been deformed too. Thus it is possible that these changes in the orbits could have generated tidal effects on the surface of the earth, like elimination of movement of sea waters or oceans (moon-earth system), or even climatological changes on earth (earth-sun system). We discuss how a pressure singularity could have been generated by an $F(R)$ gravity, how this pressure singularity could have affected the effective gravitational constant. Also, following the analysis and presentation of [6], we explicitly show in a general relativistic (GR) framework how elliptic trajectories are deformed when the Universe passes through a pressure singularity.

For this work we shall assume that the geometric background is described by a flat Friedmann-Robertson-Walker (FRW) metric, with its line element being,

$$
d s^{2}=-d t^{2}+a(t)^{2} \sum_{i=1,2,3}\left(d x^{i}\right)^{2}
$$

where $a(t)$ is the scale factor, while the Ricci scalar for the FRW metric is

$$
R=6\left(\dot{H}+2 H^{2}\right)
$$

where $H=\frac{\dot{a}}{a}$, denotes as usual the Hubble rate. 


\section{THE PERSPECTIVE OF A PRESSURE SINGULARITY IN THE NEAR PAST AND THE $F(R)$ GRAVITY PICTURE}

Following the argument of Ref. [6], it is probable that the $H_{0}$ tension might be an artifact of calibration. As was shown and claimed in [6], an abrupt change of physics before $70-150$ Myrs may, can directly affect the Cepheid parameters and can eliminate the Hubble tension. Following this line of research, we shall discuss the possibility that this abrupt change in the physics of the Universe, is caused by a global finite-time cosmological singularity of sudden type. These singularities are known to be geodetically complete, and also the strong energy conditions are respected when the Universe passes through these smooth singularities. In the following we shall assume that the spacetime metric is a flat FRW metric. Let us recall the classification of finite-time cosmological singularities, which are classified as follows [7] if the time instance of the singularity is at a finite-time $t_{s}$ :

- Type I ("Big Rip") : This is a crushing type singularity, in which case, as the singularity at $t \rightarrow t_{s}$, the scale factor $a(t)$, the total effective pressure $p_{\text {eff }}$ and energy density $\rho_{\text {eff }}$, diverge, so effectively $a \rightarrow \infty, \rho_{\text {eff }} \rightarrow \infty$, and $\left|p_{\text {eff }}\right| \rightarrow \infty$ [8].

- Type II ("sudden"): This a pressure singularity, firstly studied in Refs. [9], see also [10], for which as $t \rightarrow t_{s}$, $a \rightarrow a_{s}, \rho_{\text {eff }} \rightarrow \rho_{s},\left|p_{\text {eff }}\right| \rightarrow \infty$. This is the type of cosmological singularity for which we shall be interested for in this paper.

- Type III : In this case as $t \rightarrow t_{s}$, we have $a \rightarrow a_{s}, \rho_{\text {eff }} \rightarrow \infty,\left|p_{\text {eff }}\right| \rightarrow \infty$.

- Type IV : This type of cosmological singularity is the mildest singularity studied thoroughly in [7, 11 17], for which as $t \rightarrow t_{s}$, we have $a \rightarrow a_{s}, \rho_{\text {eff }} \rightarrow \rho_{s},\left|p_{\text {eff }}\right| \rightarrow p_{s}$, while $\frac{\mathrm{d}^{n} H}{\mathrm{~d} t^{n}}$, for $n \geq 2$ diverge.

We shall be interested for pressure singularities, or sudden singularities 9]. As we already mentioned, for these singularities, the total effective energy density, the scale factor and its first time derivative, and the Hubble rate, are finite, as the singularity is approached. However, the first derivative of the Hubble rate, the pressure and the second derivative of the scale factor are divergent as the singularity is approached. In order to have a more quantitative idea on the evolution of the Universe near a pressure singularity, let us assume that the scale factor has the following form,

$$
a(t) \simeq g(t)\left(t-t_{s}\right)^{\alpha}+f(t)
$$

where $g(t)$ and $f(t)$ and all their higher order derivatives with respect to the cosmic time are smooth functions of the cosmic time. For consistency in order not to have complex values before and after the singularity, let us assume that $a=\frac{2 m}{2 n+1}$. According to the values of the exponent $\alpha$, the following types of singularities may occur,

- For $\alpha<0$ the Universe develops a Type I singularity.

- For $0<\alpha<1$ the Universe develops a Type III singularity.

- For $1<\alpha<2$ the Universe develops a Type II singularity.

- For $2<\alpha$ the Universe develops a Type IV singularity.

Thus the pressure singularity occurs for $1<\alpha<2$, since the second derivative of the scale factor near the singularity is $\ddot{a} \sim(\alpha-1) \alpha g(t)\left(t-t_{s}\right)^{\alpha-2}$, and the same applies for the derivative of the Hubble rate, which near the singularity becomes at leading order $\dot{H} \sim \frac{(\alpha-1) \alpha\left(t-t_{s}\right)^{\alpha-2}}{f(t)}$.

For pressure singularities, the only physical quantity that diverges is the pressure, thus the energy density remains finite. The singularity occurs in a global way, so at $t=t_{s}$ which is a spacelike hypersurface, the pressure diverges. However, a pressure singularity does not lead to geodesics incompleteness, since for all causal geodesics, at $t=t_{s}$, the following integral is finite [18],

$$
\int_{0}^{\tau} d t R_{0 j 0}^{i}(t) .
$$

Regarding the strong energy conditions, these are not violated at a pressure singularity, but we have $\rho_{\text {eff }}+3 p_{\text {eff }}>0$ and $\rho_{\mathrm{eff}}>0$. This condition can be realized by many GR cosmological frameworks, like from scalar fields and cosmological fluids. In this article, for the moment, we shall assume that the scale factor $a(t)$ of Eq. (3) is realized by $F(R)$ gravity. In fact it is much more feasible to realize the scale factor (3) and at the same time to respect the strong energy conditions $\rho_{\text {eff }}+3 p_{\text {eff }}>0$ in $F(R)$ gravity. The reason is that in the case of $F(R)$ gravity, the energy 
momentum tensor receives a geometric contribution, but let us give some details on this. Consider the $F(R)$ gravity action in the presence of matter fluids,

$$
\mathcal{S}=\frac{1}{2 \kappa^{2}} \int \mathrm{d}^{4} x \sqrt{-g} F(R)+S_{m}\left(g_{\mu \nu}, \Psi_{m}\right)
$$

where $\kappa^{2}=8 \pi G$ and also $S_{m}$ denotes the matter fluids present. Note that future singularities in $F(R)$ gravity were thoroughly studied in [19]. In the metric formalism, the field equations read,

$$
R_{\mu \nu}-\frac{1}{2} R g_{\mu \nu}=\frac{\kappa^{2}}{F_{R}(R)}\left(T_{\mu \nu}+\frac{1}{\kappa^{2}}\left(\frac{F(R)-R F_{R}(R)}{2} g_{\mu \nu}+\nabla_{\mu} \nabla_{\nu} F_{R}(R)-g_{\mu \nu} \square F_{R}(R)\right)\right) .
$$

where, $F_{R}(R)=\partial F(R) / \partial R$ and $T_{\mu \nu}$ stands for the energy momentum tensor of the perfect matter fluids that are present. We can write the $F(R)$ gravity field equations in the Einstein-Hilbert form, ,

$$
R_{\mu \nu}-\frac{1}{2} R g_{\mu \nu}=T_{\mu \nu}^{m}+T_{\mu \nu}^{c u r v}
$$

where $T_{\mu \nu}^{m}$ is equal to,

$$
T_{\mu \nu}^{m}=\frac{1}{\kappa} \frac{T_{\mu \nu}}{F_{R}(R)},
$$

and originating from ordinary matter perfect fluids, while $T_{\mu \nu}^{c u r v}$ is equal to,

$$
T_{\mu \nu}^{c u r v}=\frac{1}{\kappa}\left(\frac{F(R)-R F_{R}(R)}{2} g_{\mu \nu}+F_{R}(R)^{; \mu \nu}\left(g_{\alpha \mu} g_{\beta \nu}-g_{\alpha \beta} g_{\mu \nu}\right)\right) \text {, }
$$

The energy momentum tensor (9) is thus the contribution of $F(R)$ gravity to the total energy momentum tensor. In order to further see this for a flat FRW metric (1), we consider the $f(R)$ gravity field equations for a FRW metric, which are

$$
\begin{aligned}
3 H^{2} F_{R} & =\frac{R F_{R}-F}{2}-3 H \dot{F}_{R}+\kappa^{2}\left(\rho_{r}+\rho_{m}\right), \\
-2 \dot{H} F & =\ddot{F}_{R}-H \dot{F}_{R}+\frac{4 \kappa^{2}}{3} \rho_{r},
\end{aligned}
$$

where $\rho_{m}$ and $\rho_{r}$ denote the total energy density and pressure of the dark matter and radiation fluids present, and accordingly $p_{r}$ is the radiation fluid pressure. Rewriting the field equations in the Einstein-Hilbert form, we have,

$$
\begin{aligned}
& 3 H^{2}=\kappa^{2} \rho_{t o t}, \\
& -2 \dot{H}=\kappa^{2}\left(\rho_{t o t}+P_{t o t}\right),
\end{aligned}
$$

where $\rho_{t o t}=\rho_{m}+\rho_{G}+\rho_{r}$ stands for the total energy density, $\rho_{m}$ is dark matter energy density, and $\rho_{r}$ is the radiation energy density. Finally, $\rho_{G}$ is the energy density contribution of the $F(R)$ gravity, which is defined as,

$$
\kappa^{2} \rho_{G}=\frac{F_{R} R-F}{2}+3 H^{2}\left(1-F_{R}\right)-3 H \dot{F}_{R}
$$

and its pressure is,

$$
\kappa^{2} P_{G}=\ddot{F}_{R}-H \dot{F}_{R}+2 \dot{H}\left(F_{R}-1\right)-\kappa^{2} \rho_{G}
$$

Thus the strong energy conditions near a sudden pressure singularity can be easily satisfied by an $F(R)$ gravity, and at the same time the scale factor (3) can be realized by the same $F(R)$ gravity in a consistent and easy way.

Now let us turn to the physical implications of a pressure singularity in a FRW Universe described by $F(R)$ gravity. Firstly, the divergence of the second time derivative of the scale factor implies a sudden change in the acceleration of the Universe at the singularity. The Universe may thus superaccelerate or superdecelerate for a small amount of time before and after the singularity, depending on the specific form of the scale factor. This change is an abrupt change in the physics, and in principle can be aligned with the arguments of [6] for abrupt changes in the physics before $70-150$ Myrs. 
More importantly, a pressure singularity may directly affect the effective gravitational constant in $F(R)$ gravity. In the sub-horizon approximation where the wavenumbers of the modes satisfy,

$$
\frac{k^{2}}{a^{2}} \gg H^{2}
$$

the matter density perturbations quantified by the parameter $\delta=\frac{\delta \varepsilon_{m}}{\varepsilon_{m}}$, satisfy,

$$
\ddot{\delta}+2 H \dot{\delta}-4 \pi G_{e f f}(a, k) \varepsilon_{m} \delta=0,
$$

where $G_{\text {eff }}(a, k)$ stands for the effective gravitational constant of gravity $F(R)$ theory which is defined as [20],

$$
G_{e f f}(a, k)=\frac{G}{F_{R}(R)}\left[1+\frac{\frac{k^{2}}{a^{2}} \frac{F_{R R}(R)}{F_{R}(R)}}{1+3 \frac{k^{2}}{a^{2}} \frac{F_{R R}(R)}{F_{R}(R)}}\right],
$$

where $G$ is Newton's gravitational constant. Also $\varepsilon_{m}$ in the definition of the matter density perturbation parameter $\delta$, is the total energy density of the dark matter perfect fluid. Obviously, the presence of a pressure singularity in the evolution of the Universe will directly affect the terms in the effective gravitational constant (16) which are related with the $F(R)$ function and its derivatives with respect to the Ricci scalar. This is due to the fact that the Ricci scalar in a flat FRW spacetime is of the form $R=12 H^{2}+6 \dot{H}$, and $\dot{H}$ is singular at the time instance that the pressure singularity occurs. Thus, this abrupt change in the effective gravitational constant can also affect the Cepheid parameters, thus a pressure singularity in the near past may be responsible for the abrupt change of physics in the near past. In fact, as was also pointed out in Ref. [5], a change in the effective gravitational constant at small redshifts, may remedy the $H_{0}$ tension.

So far in this article, we discussed the implications of a pressure singularity in the evolution of the Universe in the near past in a theoretical way. In this way, it is not so apparent how a pressure singularity in the near past evolution of the Universe, may actually be identified as a mean to remedy the $H_{0}$ tension. It is thus vital to have a way to identify such a scenario in an direct or indirect experimental way. Such a proposal may be realized by considering the changes on the surface of earth for cosmic times in the range 70-150 Myrs. The reason is simple, if a sudden pressure singularity occurred in the near past in our Universe, then it would affect the tidal forces of gravitationally bound objects and it would certainly disturb their orbits. Such a scenario was considered thoroughly in Ref. [6], and it was shown that a sudden pressure singularity can indeed disturb the orbits of bound objects. If this scenario indeed took place in the Universe, it would also affect the solar system, and of course it would have direct implications on the orbit of the moon around earth. Specifically, it would change the elliptic orbit of the moon around the earth and this in effect would generate observable effects on the surface of the earth. These effects would be the same in origin to the effects of the tidal gravitational forces on the surface of the earth. The most known effect of these tidal forces is the tide in the sea water. Thus a pressure singularity before $70-150 \mathrm{Myrs}$, may actually cause entire seas to disappear from the surface of the earth, if the tidal forces caused by the change in the moon's orbit were significant enough. Intriguingly enough, the changes in Tethys Sea chronologically occurred nearly $70-150 \mathrm{Myrs}$, so it is possible that the change in the orbit of the moon around earth, or even the changes in the trajectory of earth around the sun, gave rise to tidal and climate phenomena before $70-150$ Myrs. In order to have an idea how a pressure singularity could have affected the trajectory of bound objects, we shall use the paradigm of Ref. [6], which was performed in the context of simple GR. We also adopt the same GR approach in order to make the argument independent from models of $F(R)$ gravity. In Ref. [6] , the initial trajectory was considered to be a circle, we shall assume that the initial curve is actually an ellipsis. Following Ref. [6], see also [21], and also adopting their notation, in a FRW Universe in the context of GR, the energy density and pressure are $\rho(t)=\frac{3}{8 \pi G}\left(\frac{\dot{a}^{2}}{a^{2}}\right)$ and $p(t)=\frac{1}{8 \pi G}\left(2 \frac{\ddot{a}}{a}+\frac{\dot{a}^{2}}{a^{2}}\right)$, thus the pressure becomes singular for a sudden singularity. Assuming for simplicity that the pressure singularity occurs at $t=0$ a scale factor which can model a Type II singular evolution is the following,

$$
a(t)=1+c|t|^{\eta} .
$$

The pressure singularity occurs for $1<\eta<2$, and this is the case we shall consider in this presentation too. Now, the metric that describes the Newtonian limit of a spacetime near a gravitational source $M$, in a flat FRW expanding Universe is [6, 21],

$$
d s^{2}=\left(1-\frac{2 G M}{a(t) \rho}\right) \cdot d t^{2}-a(t)^{2} \cdot\left(d \rho^{2}+\rho^{2} \cdot\left(d \theta^{2}+\sin ^{2} \theta d \varphi^{2}\right)\right)
$$



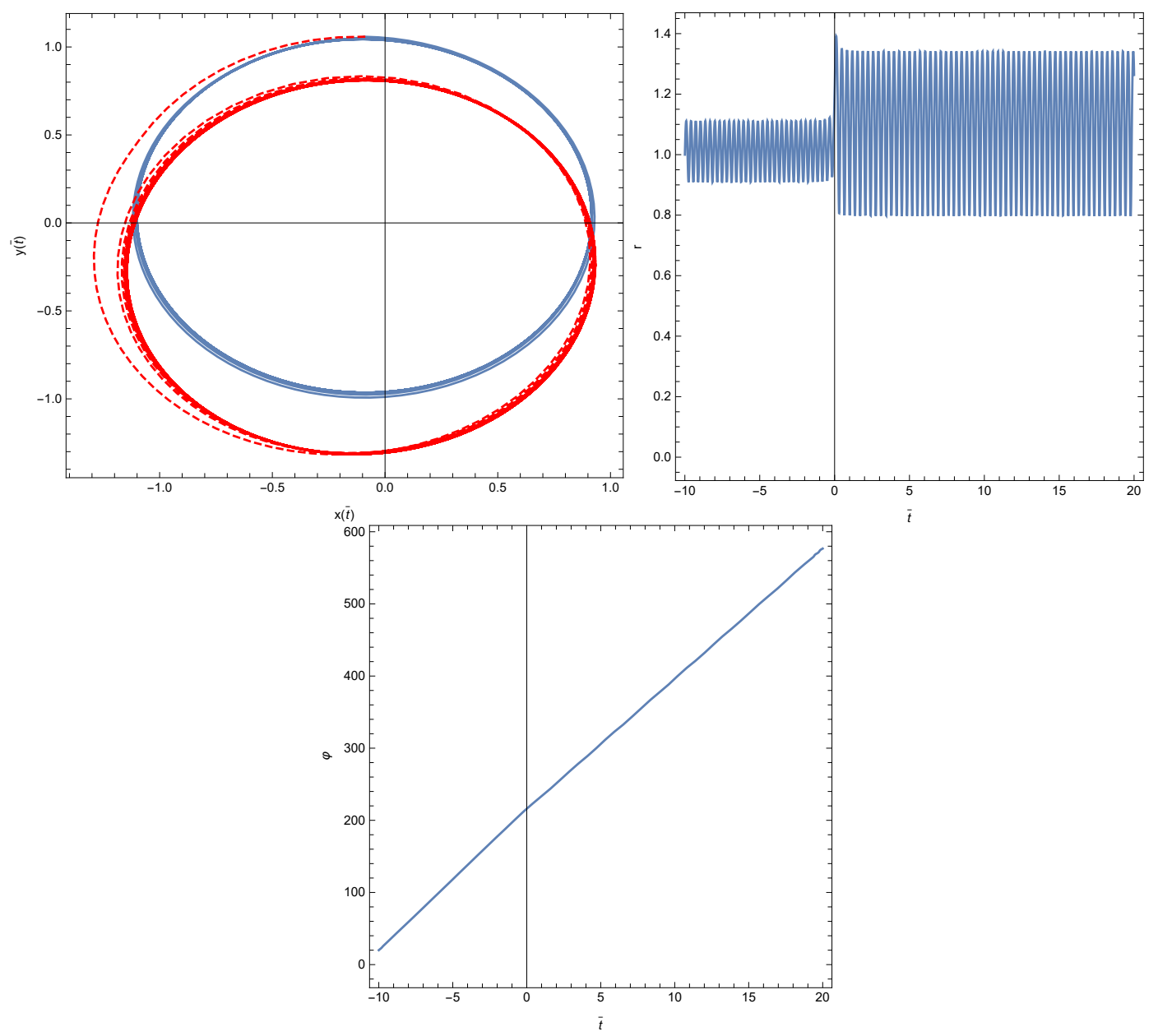

FIG. 1: Upper left plot: The trajectory of the point particle in Cartesian coordinates around the gravitational source $M$, before (blue curves), during and after (red curves) the Universe experiences the pressure singularity. Upper right plot: The radial coordinate $\bar{r}(t)$ as a function of the rescaled cosmic time. It can clearly be seen that the characteristics of the elliptic trajectory are affected by the pressure singularity at $\bar{t}=0$

which is valid when $\frac{2 G M}{a(t) \rho} \ll 1$. This condition cannot be violated in pressure singularities, since the scale factor is finite on these singularities. Replacing $r=a(t) \cdot \rho$, the geodesics equation for the metric (18) are [6, 21],

$$
\left(\ddot{r}-\frac{\ddot{a}}{a} r\right)+\frac{G M}{r^{2}}-r \dot{\varphi}^{2}=0, r^{2} \dot{\varphi}=L
$$

for both the coordinates, and $L$ is the angular momentum per unit mass, which is a conserved quantity. Combining equations (19), the radial equation of motion of the a point particle around the gravitational source $M$ is [6, 21],

$$
\ddot{r}=\frac{\ddot{a}}{a} r+\frac{L^{2}}{r^{3}}-\frac{G M}{r^{2}},
$$

and in order to obtain dimensionless quantities, one needs to make the rescalings $[6,21] \bar{r} \equiv \frac{r}{r_{0}}, \overline{\omega_{0}} \equiv \omega_{0} t_{0}$ and $\bar{t} \equiv \frac{t}{t_{0}}$, where $r_{0}$ and $t_{0}$ are arbitrary time and length scales. Upon the rescaling and also by defining $\dot{\varphi}\left(t_{0}\right)=\omega_{0} \equiv \frac{G M}{r_{0}^{3}}$, the geodesics equation becomes [6, 21],

$$
\ddot{\bar{r}}-\frac{\bar{\omega}_{0}^{2}}{\bar{r}^{3}}+\frac{\bar{\omega}_{0}^{2}}{\bar{r}^{2}}-\frac{\ddot{a}}{a} \bar{r}=0 .
$$

Hence, if the scale factor approaches a pressure singularity, it will be of the form given in Eq. (17), so the geodesics equation becomes,

$$
\ddot{\bar{r}}=\frac{\bar{\omega}_{0}^{2}}{\bar{r}^{3}}-\frac{\bar{\omega}_{0}^{2}}{\bar{r}^{2}}+\frac{c \eta(\eta-1)|\bar{t}|^{\eta-2}}{\left(c|\bar{t}|^{\eta}+1\right)} \bar{r}
$$


We solved numerically the above differential equation for various values of the parameter $\eta$ and $c$ focusing on values for which the bound system is not destroyed, but is simply disrupted. We chose the initial conditions in such a way so that the trajectory of the test particle around the gravitational source $M$ before the singularity was an ellipsis. The results of our analysis are presented in Fig. 1. In the upper left plot of Fig. 1 we present the trajectory of the point particle in Cartesian coordinates around the gravitational source $M$, before, during and after the Universe experiences the pressure singularity. As it can be seen in blue curves, the trajectory of the point test particle before the singularity was an ellipsis, and as the singularity is approached, the trajectory is disrupted, starting at nearly $t=0$. At that point the red curve describes the disruption of the trajectory of the point particle around $M$, which after a while it also becomes an ellipsis. In the upper right plot of Fig. [1 we plot the radial coordinate as a function of the rescaled cosmic time. As it can be seen, the elliptic trajectory is changed at the singularity, and also the trajectory is an elliptic curve after the singularity, with different characteristics though. In the bottom of Fig. 1 we plot the function $\varphi(t)$. These simple considerations can be extended in the $F(R)$ gravity case, for specific models of $F(R)$ gravity, but they suffice to support our argument that pressure singularities might actually have caused observable tidal or even climatological effects on earth before $70-150$ Myrs. These effects might changed the surface of the earth, removing entire seas or even oceans from their initial position, if the tidal effects of moon's gravity were severe enough.

\section{CONCLUSIONS}

In this article we considered the scenario that an abrupt change in physics occurred around $70-150$ Myrs ago might have been caused by a pressure singularity. The Universe can smoothly pass through such a pressure singularity, since these are not crushing type singularities, and the only physical quantity that diverges is the effective pressure of the Universe everywhere on the three dimensional spacelike hypersurface corresponding to the time instance at which the singularity occurs. The strong energy conditions are not violated in such types of singularities, and we explained how such singularities might occur in the context of $F(R)$ gravity. Also in $F(R)$ gravity, the strong energy conditions can easily be satisfied. Moreover, we showed that a pressure singularity can actually cause a blow-up in the $F(R)$ gravity effective gravitational constant, and this phenomenon is also aligned with the abrupt physics changes argument of Refs. [5]. We should also note that there might be a connection of our present approach with a maximal turn round radius which is related to the shape of the specific $f(R)$ gravity, see for example 22]. Apart from this feature which may depend strongly on the specific $f(R)$ gravity model, there is a similarity between the pressure singularity and the transition redshift that relates deceleration to acceleration in cosmographic approaches [23]. Certainly, these perspectives should be closely discussed in some future article.

Coming back on the results of our article, as we discussed in the text, a pressure singularity occurring before 70 - 150 Myrs, could have disrupted the elliptic orbits of bound objects in the Universe, without destroying them, but deforming these to ellipses with different characteristics. As we showed numerically, this is feasible in the presence of a pressure singularity. Such deformation in the elliptic trajectories of bound objects could also have occurred in our solar system, and could have had direct effects on the surface of the earth or even in the climate of the earth before $70-150$ Myrs. Indeed, even deforming the orbit of the moon could have had observable effects on oceans and sea waters at that time. Also the same effects along with climatological changes could have occurred due to the change of the elliptic orbit of the earth around the sun. Thus earth's primordial history might reveal such abrupt changes in the physics of the Universe, as is also nicely pointed out in Ref. [6]. In the same line of research, recently in Ref. 24] it was also pointed out that the extinction of dinosaurs may be related to the Hubble crisis. This argument further supports the general idea of seeking imprints of the late-time Universe on the geological and environmental history of earth. This line of research is sensational, it might open up new horizons in theoretical cosmology and astrophysics.

\section{Acknowledgments}

We are grateful to Leandros Perivolaropoulos for his encouraging comments and suggestions. This work was supported by MINECO (Spain), project PID2019-104397GB-I00 (S.D.O).

[1] L. Perivolaropoulos and F. Skara, arXiv:2105.05208 [astro-ph.CO]].

[2] L. Verde, T. Treu and A. G. Riess, Nature Astron. 3, 891 doi:10.1038/s41550-019-0902-0 [arXiv:1907.10625] [astro-ph.CO]].

[3] E. Mortsell, A. Goobar, J. Johansson and S. Dhawan, arXiv:2105.11461 [astro-ph.CO]]. 
[4] L. Perivolaropoulos and F. Skara, Phys. Rev. D 104 (2021) no.12, 123511 doi:10.1103/PhysRevD.104.123511 arXiv:2109.04406 [astro-ph.CO]].

[5] V. Marra and L. Perivolaropoulos, Phys. Rev. D 104 (2021) no.2, L021303 doi:10.1103/PhysRevD.104.L021303 arXiv:2102.06012 [astro-ph.CO]].

[6] L. Perivolaropoulos, Phys. Rev. D 94 (2016) no.12, 124018 doi:10.1103/PhysRevD.94.124018 arXiv:1609.08528 [gr-qc]].

[7] S. Nojiri, S. D. Odintsov and S. Tsujikawa, Phys. Rev. D 71 (2005) 063004 doi:10.1103/PhysRevD.71.063004 hep-th/0501025.

[8] R. R. Caldwell, M. Kamionkowski and N. N. Weinberg, Phys. Rev. Lett. 91, 071301 (2003) arXiv:astro-ph/0302506.;

B. McInnes, JHEP 0208 (2002) 029 arXiv:hep-th/0112066;

S. Nojiri and S. D. Odintsov, Phys. Lett. B 562, 147 (2003) arXiv:hep-th/0303117;

V. Gorini, A. Kamenshchik and U. Moschella, Phys. Rev. D 67 (2003) 063509 astro-ph/0209395;

M. Cataldo, L. P. Chimento and M. G. Richarte, Phys. Rev. D 95 (2017) no.6, 063510;

L. P. Chimento and M. G. Richarte, Phys. Rev. D 93 (2016) no.4, 043524 [erratum: Phys. Rev. D 95 (2017) no.6, 069902] doi:10.1103/PhysRevD.93.043524 arXiv:1512.02664 [gr-qc]]. ;

E. Elizalde, S. Nojiri and S. D. Odintsov, Phys. Rev. D 70 (2004) 043539 hep-th/0405034. ;

V. Faraoni, Int. J. Mod. Phys. D 11, 471 (2002) arXiv:astro-ph/0110067;

P. Singh, M. Sami and N. Dadhich, Phys. Rev. D 68, 023522 (2003) arXiv:hep-th/0305110;

P. X. Wu and H. W. Yu, Nucl. Phys. B 727, 355 (2005) arXiv:astro-ph/0407424;

M. Sami and A. Toporensky, Mod. Phys. Lett. A 19, 1509 (2004) arXiv:gr-qc/0312009];

H. Stefancic, Phys. Lett. B 586, 5 (2004) arXiv:astro-ph/0310904;

L. P. Chimento and R. Lazkoz, Phys. Rev. Lett. 91, 211301 (2003) arXiv:gr-qc/0307111;

Mod. Phys. Lett. A 19, 2479 (2004) arXiv:gr-qc/0405020];

J. G. Hao and X. Z. Li, Phys. Lett. B 606, 7 (2005) arXiv:astro-ph/0404154];

E. Babichev, V. Dokuchaev and Yu. Eroshenko, Class. Quant. Grav. 22, 143 (2005) arXiv:astro-ph/0407190;

X. F. Zhang, H. Li, Y. S. Piao and X. M. Zhang, Mod. Phys. Lett. A 21, 231 (2006) arXiv:astro-ph/0501652;

F. S. N. Lobo, Phys. Rev. D 71, 084011 (2005) arXiv:gr-qc/0502099;

R. G. Cai, H. S. Zhang and A. Wang, Commun. Theor. Phys. 44, 948 (2005) arXiv:hep-th/0505186;

M. P. Dabrowski, C. Kiefer and B. Sandhofer, Phys. Rev. D 74, 044022 (2006) arXiv:hep-th/0605229

[9] J. D. Barrow, Class. Quant. Grav. 21 (2004) L79 gr-qc/0403084. ;

S. Nojiri and S. D. Odintsov, Phys. Lett. B 595 (2004) 1 doi:10.1016/j.physletb.2004.06.060 hep-th/0405078.

[10] J. D. Barrow and C. G. Tsagas, Class. Quant. Grav. 22, 1563 (2005) arXiv:gr-qc/0411045;

L. Fernandez-Jambrina and R. Lazkoz, Phys. Rev. D 70, 121503 (2004) arXiv:gr-qc/0410124;

M. Bouhmadi-Lopez, P. F. Gonzalez-Diaz and P. Martin-Moruno, Phys. Lett. B 659, 1 (2008) arXiv:gr-qc/0612135;

J. D. Barrow and S. Z. W. Lip, arXiv:0901.1626 [gr-qc];

M. Bouhmadi-Lopez, Y. Tavakoli and P. V. Moniz, arXiv:0911.1428 [gr-qc].;

J. D. Barrow, A. B. Batista, J. C. Fabris, M. J. S. Houndjo and G. Dito, Phys. Rev. D 84 (2011) 123518 arXiv:1110.1321 $[\mathrm{gr}-\mathrm{qc}]]$

M. Bouhmadi-Lopez, C. Kiefer and M. Kramer, Phys. Rev. D 89 (2014) 6, 064016 [arXiv:1312.5976 [gr-qc]];

M. Bouhmadi-Lopez, P. Chen and Y. W. Liu, Eur. Phys. J. C 73 (2013) 2546 arXiv:1302.6249 [gr-qc]].

[11] S. Nojiri and S. D. Odintsov, Phys. Rev. D 70 (2004) 103522 doi:10.1103/PhysRevD.70.103522 hep-th/0408170].

[12] J. D. Barrow and A. A. H. Graham, Phys. Rev. D 91, no. 8, 083513 (2015) arXiv:1501.04090 [gr-qc]].

[13] S. Nojiri, S. D. Odintsov and V. K. Oikonomou, Phys. Rev. D 91 (2015) no.8, 084059 doi:10.1103/PhysRevD.91.084059 arXiv:1502.07005 [gr-qc]].

[14] S. Nojiri, S. D. Odintsov, V. K. Oikonomou and E. N. Saridakis, JCAP 1509 (2015) 044 doi:10.1088/1475-7516/2015/9/044, 10.1088/1475-7516/2015/09/044 arXiv:1503.08443 [gr-qc]].

[15] S. D. Odintsov and V. K. Oikonomou, Phys. Rev. D 92 (2015) no.2, 024016 doi:10.1103/PhysRevD.92.024016 arXiv:1504.06866 [gr-qc]].

[16] V. K. Oikonomou, Phys. Rev. D 92 (2015) no.12, 124027 doi:10.1103/PhysRevD.92.124027 arXiv:1509.05827 [gr-qc]].

[17] V. K. Oikonomou, Int. J. Geom. Meth. Mod. Phys. 13 (2016) no.03, 1650033 doi:10.1142/S021988781650033X arXiv:1512.04095 [gr-qc]].

[18] L. Fernandez-Jambrina and R. Lazkoz, Phys. Rev. D 70 (2004), 121503 doi:10.1103/PhysRevD.70.121503 arXiv:gr-qc/0410124 [gr-qc]].

[19] S. Nojiri and S. D. Odintsov, eConf C0602061 (2006), 06 doi:10.1142/S0219887807001928 arXiv:hep-th/0601213 [hep-th]].

[20] K. Bamba, A. Lopez-Revelles, R. Myrzakulov, S. D. Odintsov and L. Sebastiani, Class. Quant. Grav. 30 (2013), 015008 doi:10.1088/0264-9381/30/1/015008 arXiv:1207.1009 [gr-qc]].

[21] S. Nesseris and L. Perivolaropoulos, Phys. Rev. D 70 (2004), 123529 doi:10.1103/PhysRevD.70.123529 arXiv:astro-ph/0410309 [astro-ph]].

[22] S. Capozziello, K. F. Dialektopoulos and O. Luongo, Int. J. Mod. Phys. D 28 (2018) no.03, 1950058 doi:10.1142/S0218271819500585 arXiv:1805.01233 [gr-qc]].

[23] S. Capozziello, O. Farooq, O. Luongo and B. Ratra, Phys. Rev. D 90 (2014) no.4, 044016 doi:10.1103/PhysRevD.90.044016 arXiv:1403.1421 [gr-qc]].

[24] L. Perivolaropoulos, arXiv:2201.08997 [astro-ph.EP]]. 